\title{
OPTIMAL HEAT INPUT FOR ESTIMATING LUIKOV'S PARAMETERS IN A HEAT AND MASS TRANSFER PROBLEM
}

\author{
Abdelmalek Bensefia ${ }^{1,2}$, Mohammed Boussaid ${ }^{2}$, and \\ Tahar Loulou ${ }^{1}$ \\ ${ }^{1}$ Université de Bretagne Sud, Centre de Recherche, LIMATB, \\ Lorient, France \\ ${ }^{2}$ Faculté des Sciences de l'Ingénieur, Département Énergétique, \\ Université M'hamed Bouguara, Boumerdes, Algeria
}

In this article, the problem of computing an optimal heat input in Luikov's heat and mass transfer problem is detailed and analyzed. The main objective is the establishment of an optimal timedependent heat flux profile with the goal of maximizing the temperature and moisture sensitivities of some parameters to this excitation in a drying process. Such maximization makes the estimation of the desired parameters possible, easier, and with lim-ited uncertainty intervals. It also helps to reduce the linearity dependence between the para-meters of interest and the number of temperature and moisture sensors used. The estimation of the optimal heat input is obtained with Uzawa's algorithm, while the estimation of para-meters is performed with Levenberg-Marquardt's method of minimization of the ordinary least-square criterion. The six dimensionless parameters characterizing Luikov's equations are estimated successfully with this optimal heat flux profile, which also helps to reduce the number of both temperature and moisture sensors needed in the estimation procedure. By doing so, the objective of estimating simultaneously the six parameters which appear in the formulation of Luikov's physical problem is reached by using a limited transient temperature and/or moisture measurements taken anywhere in the drying medium.

\section{INTRODUCTION}

Sustainable development and energy conservation requires a constant search for new materials which are very efficient in terms of thermal and mass insulation, with high mechanical performance and especially easy recycling without affecting the environment at end of life. In this context, our laboratory is conducting research activities on the heat and mass transfer in a series of earth-plant-based concretes intended mainly for the construction of ecological habitat. Heat and mass transfer phenomena are omnipresent in all the life cycles of these construction materials, i.e., during processing, for everyday use, and last, in recycling processes. Thus, the investigation of the phenomena of coupled heat and mass transfer in capillary porous media is an important topic of research investigation.

Address correspondence to Tahar Loulou, Université de Bretagne Sud, Centre de Recherche, LET2E, Rue Saint Maude F-56321, Lorient Cedex, France. E-mail: tahar.loulou@univ-ubs.fr 


\begin{tabular}{|llll|}
\hline \multicolumn{3}{|c|}{ NOMENCLATURE } \\
$a, a_{q}$ & thermal diffusivity & $t$ & time \\
$a_{m}$ & moisture diffusivity & $T(x, t)$ & temperature field \\
$\mathrm{Bi}_{m}$ & moisture Biot number & $\Delta T(x, t)$ & variation temperature field \\
$\mathrm{Bi}_{q}$ & heat Biot number & $x$ & space coordinates \\
$c_{m}$ & specific moisture & $\beta$ & Lagrange multiplier \\
$c_{q}$ & specifi cheat & $\delta$ & thermo-gradient coefficient \\
$C(x, t)$ & moisture field & $\varepsilon$ & phase conversion factor \\
$\Delta C(x, t)$ & variation moisture field & $\varepsilon$ & small number \\
$h_{m}$ & mass transfer coefficient & Subscripts & \\
$h_{q}$ & heat transfer coefficient & $a$ & air \\
$k, k_{q}$ & thermal conductivity & $f$ & final \\
$k_{m}$ & moisture conductivity & $m$ & moisture \\
$\mathrm{Ko}$ & Kossovitch number & $q$ & heat \\
$L$ & thickness & $r$ & reference \\
$\mathrm{Lu}$ & Luikov number & \multicolumn{2}{c}{ Superscripts } \\
$\mathcal{L}$ & Lagrangian functional & $i$ & initial \\
$\mathrm{Pn}$ & Posnov number & + & dimensionless variable \\
$q(t)$ & applied heat flux & \multicolumn{2}{c}{} \\
\hline
\end{tabular}

The theory of heat and mass transfer is based on the phenomenon of thermodiffusion found by Luikov in 1935 [1]. On the basis of the thermodynamics of irreversible processes, he defined a system of coupled partial differential equations known as Luikov's equations. This system of equations has been used extensively in the literature to study different geometric configurations with several drying bodies. Luikov's equations are governed by six fundamental parameters which take into account the heat andmass diffusion effects in the medium and the applied boundary conditions (energy and mass balance at the boundaries). These dimensionless parameters are $\mathrm{Bi}_{q}$, the heat Biot number; $\mathrm{Bi}_{m}$, the moisture Biot number; $\mathrm{Lu}$, the Luikov number; Pn, the Posnov number; Ko, the Kossovitch number; and $\varepsilon$, the phase conversion factor.

Recently, several articles dealing with the solution of the inverse problem of coupled heat and mass transfer have appeared in the literature [2-12]. In these articles, both parameter and/or function estimation approaches are used in the solution of these inverse problems by using temperature and/or moisture measurements. The most-used methods are the Levenberg-Marquardt method in parameter estimation and the conjugate gradient method in function estimation, respectively. For example, for the function estimation approach, Huang et al. [13] and Saker et al. [8-10] used the conjugate gradient method to determine the unknown time-dependentand spacewise variations of mass and heat transfer coefficients in porous material from the knowledge of the temperature and moisture measurements taken within a drying body.

The extensive work of Kanevce and his co-workers [2-7] was based mainly on finding the possibilities of estimating simultaneously the moisture diffusivity, together with other thermophysical properties of vegetables, as well as the heat and mass transfer coefficients. Their method requires a single drying experiment and a single temperature measurement probe. As a representative drying vegetable product, thin slices of potato have been used as the sample material. An analysis 
of the influence of the drying air velocity, temperature and relative humidity, drying body dimensions, and drying time on the moisture diffusivity estimation enables them to predict the optimal design condition of appropriate experiments to be conducted as well.

In their article, Dantas et al. [14] estimated three parameters among the six parameters characterizing Luikov's system by using only one temperature sensor. Later, the estimation of four parameters was attempted and succeeded by using simultaneously the measurement of two sets of temperature and moisture sensors with different locations for each set [15]. In these two articles, substantial work was accomplished toward optimizing the experimental conditions leading to the best estimate of a maximum of parameters among the six presents in Luikov's formulation with a reduced amount of uncertainties. Their work concentrated mainly on the determination of the minimum temperature and moisture sensors and the optimal duration of the experiment needed in the estimation algorithm. The results presented concern the drying process of four test cases: tests 1-3 represent a commonly used wood, while test 4 deals with the drying of ceramics.

The objective of the inverse problem, presented in [14, 15], was the estimation of the dimensionless parameters $\mathrm{Bi}_{q}, \mathrm{Bi}_{m}, \mathrm{Lu}, \mathrm{Pn}, \mathrm{Ko}$, and $\varepsilon$ from the temperature and moisture measurements. As the sensitivity coefficients of Pn and $\varepsilon$ were practically null, it was impossible to estimate these two parameters despite great efforts involved in optimizing the experiment conditions.

The attempt at estimating Luikov's parameters from an experiment, in which the postions, the number of sensors of temperature and/or moisture, and the experiment duration were optimized, did not reached its target; i.e., the simultaneous estimation of the six parameters from a single experiment failed. Indeed, the optimal design involving the choice of the states to be measured, the frequency and location of measurements, and the duration of experiments shows its limits; thus, searching for another solution to this problem is the obvious course to take. The estimation of Luikov's parameters certainly depends on the above-mentioned factors and on the applied boundary conditions as well. This fact suggests that we may form some hypotheses on the experimental conditions used until now, and attempt to optimize one boundary condition of the direct problem which makes (1) the identifiability of all parameters possible, and (2) the estimation of these parameters with the best precision.

In this work, we consider the problem of the determination of an optimal input (one boundary condition) for a nonlinear distributed parameter system with the following given experimental conditions: (1) the number of sensors is known and limited; (2) the sensor locations are well known; (2) the experiment duration is set; and (4) the amplitude of unknown thermal input is constrained. The article is organized in four sections including the present one (introduction). Section two presents in detail the formulation and derivation of the problem of finding an optimal heat input with constraint. Section 3 presents the results of two analyzed test cases: ceramics and wood. The last section presents the main conclusions of this research.

\section{PROBLEM FORMULATION}

In this article, we consider the problem of computing temperature and moisture distribution during contact drying of a moist, porous medium; see Figure 1. 


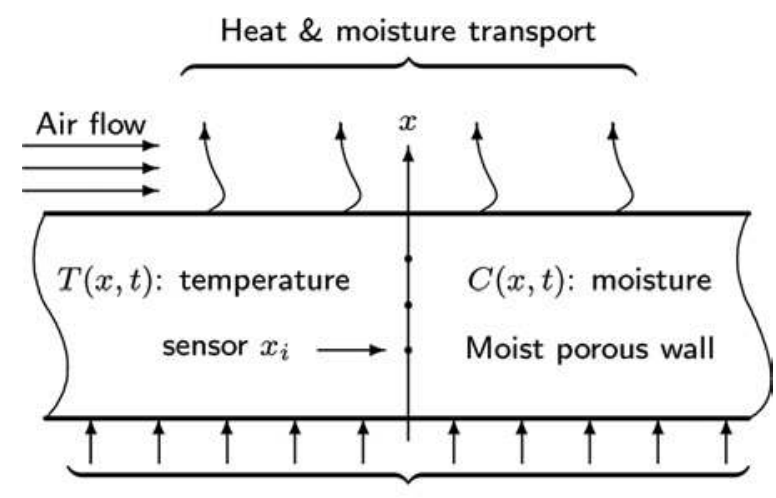

Supplied heat flux $q(t)$

Figure 1. Geometric configuration and coordinate system.

The initial theoretical formulation given in [1] is reproduced here with slight changes in the boundary conditions, which are taken as a function of time, i.e., the applied heat flux $q(t)$ at $x=0$, the surrounding air temperature, and the surrounding moisture content, at $x=L$, are all considered as time-dependent functions. In this case, the dimensionless coupled heat and mass transfer problem is given by

$$
\begin{gathered}
\frac{\partial T(x, t)}{\partial t}=\frac{\partial^{2} T(x, t)}{\partial x^{2}}-\varepsilon K o \frac{\partial C(x, t)}{\partial t} \quad 0<x<1 \quad t>0 \\
T(x, t)=0 \quad 0<x<1 \quad t=0 \\
-\frac{\partial T(x, t)}{\partial x}=q(t) \quad x=0 \quad t>0 \\
-\frac{\partial T(x, t)}{\partial x}=\mathrm{Bi}_{q}\left[T(x, t)-T_{a}(t)\right]-(1-\varepsilon) \operatorname{KoLuBi}_{m}\left[C(x, t)-C_{a}(t)\right] \quad x=1 \quad t>0
\end{gathered}
$$

for the heat transfer phenomena and

$$
\begin{gathered}
\frac{\partial C(x, t)}{\partial t}=\operatorname{Lu} \frac{\partial^{2} C(x, t)}{\partial x^{2}}-\operatorname{Lu} \operatorname{Pn} \frac{\partial^{2} T(x, t)}{\partial x^{2}} \quad 0<x<1 \quad t>0 \\
C(x, t)=0 \quad 0<x<1 \quad t=0 \\
-\frac{\partial C(x, t)}{\partial x}+\operatorname{Pn} \frac{\partial T(x, t)}{\partial x}=0 \quad x=0 \quad t>0 \\
-\frac{\partial C(x, t)}{\partial x}+\operatorname{Pn} \frac{\partial T(x, t)}{\partial x}=\operatorname{Bi}_{m}\left[C(x, t)-C_{a}(t)\right] \quad x=1 \quad t>0
\end{gathered}
$$

for the mass transfer phenomena. The source terms present in both diffusion equations and boundary conditions manifest (translate) the coupling effect between the 
two phenomena: heat and mass. The dimensionless variables appearing in the above system of equations are defined by

$\mathrm{Bi}_{m}=\frac{h_{m} L}{k_{m}} \quad$ dimensionless mass transfer coefficient

$\mathrm{Bi}_{q}=\frac{h L}{k} \quad$ dimensionless heat transfer coefficient

$\mathrm{Ko}=\frac{\lambda}{c} \frac{C_{r}}{T_{r}} \quad$ Kossovitch number

$\mathrm{Lu}=\frac{a_{m}}{a} \quad$ Luikov number or (Lewis number) $)^{-1}$

$\mathrm{Pn}=\delta \frac{T_{r}}{C_{r}} \quad$ Possnov number

$c=\frac{c_{q}}{c_{m}} \quad$ ratio of specific heat to specific moisture content

$q^{+}(t)=\frac{q(t) L}{k T_{r}} \quad$ dimensionless heat flux

$T^{+}\left(x^{+}, t^{+}\right)=\frac{T(x, t)-T^{i}}{T_{r}} \quad$ dimensionless temperature

$T_{a}^{+}\left(t^{+}\right)=\frac{T_{a}(t)-T^{i}}{T_{r}} \quad$ dimensionless air temperature

$C^{+}\left(x^{+}, t^{+}\right)=\frac{C^{i}-C(x, t)}{C_{r}} \quad$ dimensionless moisture

$C_{a}^{+}\left(t^{+}\right)=\frac{C^{i}-C_{a}(t)}{C_{r}} \quad$ dimensionless air moisture

$x^{+}=\frac{x}{L} \quad$ dimensionless coordinate

$t^{+}=\frac{a t}{L^{2}} \quad$ dimensionless time

where $T_{r}$ and $C_{r}$ are a reference temperature and reference moisture, respectively. For the sake of clarity, the superscript $(+)$ is omitted in the above-presented system of equations (1)-(8). The solution of Luikov's equations can be obtained analytically in some simple linear cases [14-19]. Equations (1)-(8) are referred to as a direct problem in the inverse problem terminology and aim to determine the dimensionless temperature and moisture fields $T(x, t)$ and $C(x, t)$.

The objective of the inverse problem, presented in [14, 15], was the estimation of dimensionless parameters $\mathrm{Bi}_{q}, \mathrm{Bi}_{m}, \mathrm{Lu}, \mathrm{Pn}, \mathrm{Ko}$, and $\varepsilon$ from the temperature and moisture measurements. As the sensitivity coefficients of $\mathrm{Pn}$ and $\varepsilon$ were practically null, it was impossible to estimate these two parameters despite the great efforts involved in optimizing the experiment conditions.

The design of optimum experiments is of great importance in parameter and function estimations in all scientific fields [20, 21]. Indeed, it is well known in the literature that the choice of experimental conditions, such as the number and location of sensors, experiment duration, etc., for distributed systems has a strong connection with the accuracy of estimated unknowns [20, 22-24]. The optimal design basically consists of examining a priori some kind of measure ofthe accuracy of the estimated parameters and functions. Many criteria are proposed in the specialized literature, and the most used among them are D-optimality criterion, E-optimality criterion, A-optimality criterion, and the sensitivity criterion. All of them are based on the concept of the Fisher information matrix (FIM), which is widely used in optimum experimental design theory $[25,26,21]$. The elements of this matrix are nothing more than the sensitivity coefficients, i.e., the derivatives of the state variables (temperature, moisture) with respect to system parameters. 
The sensitivity of temperature and moisture, as a function of time, with respect to the parameter $\varepsilon$ and $\mathrm{Pn}$ at a given sensor location $x_{i}$ are defined by [20, 22-24, 27]

$$
\begin{aligned}
T_{\varepsilon}\left(x_{i}, t\right) & =\frac{\partial T\left(x_{i}, t\right)}{\partial \varepsilon} \quad \text { and } \quad T_{p}\left(x_{i}, t\right)=\frac{\partial T\left(x_{i}, t\right)}{\partial \mathrm{Pn}} \\
C_{\varepsilon}\left(x_{i}, t\right) & =\frac{\partial C\left(x_{i}, t\right)}{\partial \varepsilon} \quad \text { and } \quad C_{p}\left(x_{i}, t\right)=\frac{\partial C\left(x_{i}, t\right)}{\partial \mathrm{Pn}}
\end{aligned}
$$

There are different ways to compute the sensitivity coefficients: (1) finitedifference method, (2) differentiation of the direct problem, and (3) the analytical method if one has at his disposal an analytical solution of the direct problem. For computational purposes, the central finite-difference method is simple and gives excellent results if the rounding errors and truncated errors are carefully handled. Applying the above definitions of the sensitivity functions to the direct problem, we obtain the following set of equations:

$$
\begin{aligned}
& \frac{\partial T_{\varepsilon}(x, t)}{\partial t}=\frac{\partial^{2} T_{\varepsilon}(x, t)}{\partial x^{2}}-\operatorname{Ko} \frac{\partial C(x, t)}{\partial t}-\varepsilon \operatorname{Ko} \frac{\partial C_{\varepsilon}(x, t)}{\partial t} \quad 0<x<1 \quad t>0 \\
& T_{\varepsilon}(x, t)=0 \quad 0<x<1 \quad t=0 \\
& \frac{\partial T_{\varepsilon}(x, t)}{\partial x}=0 \quad x=0 \quad t>0 \\
& -\frac{\partial T_{\varepsilon}(x, t)}{\partial x}=\mathrm{Bi}_{q} T_{\varepsilon}(x, t)+\mathrm{KoLuBi}_{m}\left[C(x, t)-C_{a}(t)\right] \\
& -(1-\varepsilon) \mathrm{Ko} \mathrm{LuBi}_{m} C_{\varepsilon}(x, t) \quad x=1 \quad t>0 \\
& \frac{\partial C_{\varepsilon}(x, t)}{\partial t}=\operatorname{Lu} \frac{\partial^{2} C_{\varepsilon}(x, t)}{\partial x^{2}}-\operatorname{LuPn} \frac{\partial^{2} T_{\varepsilon}(x, t)}{\partial x^{2}} \quad 0<x<1 \quad t>0 \\
& C_{\varepsilon}(x, t)=0 \quad 0<x<1 \quad t=0 \\
& \frac{\partial C_{\varepsilon}(x, t)}{\partial x}=0 \quad x=0 \quad t>0 \\
& -\frac{\partial C_{\varepsilon}(x, t)}{\partial x}+\operatorname{Pn} \frac{\partial T_{\varepsilon}(x, t)}{\partial x}=\mathrm{Bi}_{m} C_{\varepsilon}(x, t) \quad x=1 \quad t>0
\end{aligned}
$$

for the temperature and moisture sensitivities with respect to the parameter $\varepsilon$, and

$$
\begin{gathered}
\frac{\partial T_{p}(x, t)}{\partial t}=\frac{\partial^{2} T_{p}(x, t)}{\partial x^{2}}-\varepsilon \operatorname{Ko} \frac{\partial C_{p}(x, t)}{\partial t} \quad 0<x<1 \quad t>0 \\
T_{p}(x, t)=0 \quad 0<x<1 \quad t=0 \\
\frac{\partial T_{p}(x, t)}{\partial x}=0 \quad x=0 \quad t>0
\end{gathered}
$$




$$
\begin{gathered}
-\frac{\partial T_{p}(x, t)}{\partial x}=\mathrm{Bi}_{q} T_{p}(x, t)-(1-\varepsilon) \mathrm{Ko} \mathrm{LuBi}_{m} C_{p}(x, t) \quad x=1 \quad t>0 \\
\frac{\partial C_{p}(x, t)}{\partial t}=\mathrm{Lu} \frac{\partial^{2} C_{p}(x, t)}{\partial x^{2}}-\mathrm{Lu} \frac{\partial^{2} T(x, t)}{\partial x^{2}}-\operatorname{LuPn} \frac{\partial^{2} T_{p}(x, t)}{\partial x^{2}} \quad 0<x<1 \quad t>0 \\
C_{p}(x, t)=0 \quad 0<x<1 \quad t=0 \\
-\frac{\partial C_{p}(x, t)}{\partial x}+\frac{\partial T(x, t)}{\partial x}=0 \quad x=0 \quad t>0 \\
-\frac{\partial C_{p}(x, t)}{\partial x}+\frac{\partial T(x, t)}{\partial x}+\operatorname{Pn} \frac{\partial T_{p}(x, t)}{\partial x}=\operatorname{Bi}_{m} C_{p}(x, t) \quad x=1 \quad t>0
\end{gathered}
$$

for the temperature and moisture sensitivities with respect to Posnov number Pn.

Due to its simplicity, the sensitivity criterion (or T-optimality criterion), which consists of maximizing the trace of the information matrix, is used in this work to increase the sensitivity coefficients $T_{\varepsilon}\left(x_{i}, t\right)$ and $C_{\varepsilon}\left(x_{i}, t\right)$ by building an optimal heat input with the goal of estimating simultaneously all Luikov's parameters [28]. As shown in Eqs. (1)-(8), the direct problem is subjected to heat input (heating) from its bottom side, expressed by the quantity $q(t)$, and heat and mass convection losses from its top side, governed by $\left(\mathrm{Bi}_{q}, T_{a}(t)\right)$ and $\left(\mathrm{Bi}_{m}, C_{a}(t)\right)$, respectively. Any one of these boundary conditions can be used as an optimal input in this design problem, and the present article considers the heat flux $q(t)$ as the input to be optimized. The sensitivity criterion is defined by

$$
J_{1}(q)=\sum_{i=1}^{N t} \int_{0}^{t_{f}}\left[\left[T_{\varepsilon}\left(x_{i}, t\right)\right]^{2}+\left[T_{p}\left(x_{i}, t\right)\right]^{2}\right] d t+\sum_{i=1}^{N c} \int_{0}^{t_{f}}\left[\left[C_{\varepsilon}\left(x_{i}, t\right)\right]^{2}+\left[C_{p}\left(x_{i}, t\right)\right]^{2}\right] d t
$$

where $N_{t}$ is the number of temperature sensors and $N_{c}$ is the number of moisture sensors installed in the drying body; see Figure 1 . The design problem in view here consists of the maximization of the criterion $J_{1}(q)$ by searching for the best time profile of $q(t)$. Indeed, the larger the magnitude of the input $q(t)$, the greater will be the sensitivities $T_{\varepsilon}\left(x_{i}, t\right)$ and $C_{\varepsilon}\left(x_{i}, t\right)$. However, for some thermal and physical considerations the input $q(t)$ must be constrained; otherwise the maximization of Eq. (27) will yield the trivial solution that the optimal input $q(t)$ is infinite. Accordingly, the input $q(t)$ is constrained such that it is less than or equal to a constant $E$ :

$$
\int_{0}^{t_{f}} q^{2}(t) d t \leq E
$$

For some practical considerations, maximizing the criterion (27), is usually transformed into the minimization of the new following functional:

$$
J_{2}(q)=-\sum_{i=1}^{N t} \int_{0}^{t_{f}}\left[\left[T_{\varepsilon}\left(x_{i}, t\right)\right]^{2}+\left[T_{p}\left(x_{i}, t\right)\right]^{2}\right] d t-\sum_{i=1}^{N c} \int_{0}^{t_{f}}\left[\left[C_{\varepsilon}\left(x_{i}, t\right)\right]^{2}+\left[C_{p}\left(x_{i}, t\right)\right]^{2}\right] d t
$$


with the same constraint on the input $q(t)$ given in Eq. (28). The next step in this optimization problem is the computation of the optimality conditions which can be obtained by the introduction of the augmented Lagrangian functional $\mathscr{L}(q)$. Indeed, this augmented functional takes into account all the constraints under which the criterion $J_{2}(q)$ must be minimized, i.e., the constraint Eq. (28), the direct problem given in Eqs. (1)-(8), and the two sensitivity problems given by Eqs. (11)-(18) and (19)-(26). Explicitly, this can be written as

$$
\begin{aligned}
& \mathscr{L}(q)=-\sum_{i=1}^{N t} \int_{0}^{t_{f}}\left[\left[T_{\varepsilon}\left(x_{i}, t\right)\right]^{2}+\left[T_{p}\left(x_{i}, t\right)\right]^{2}\right] d t-\sum_{i=1}^{N c} \int_{0}^{t_{f}}\left[\left[C_{\varepsilon}\left(x_{i}, t\right)\right]^{2}+\left[C_{p}\left(x_{i}, t\right)\right]^{2}\right] d t \\
& +\beta \int_{0}^{t_{f}}\left[q^{2}(t)-\frac{E}{t_{f}}\right] d t+\int_{0}^{t_{f}} \int_{0}^{L}\left[\frac{\partial^{2} T(x, t)}{\partial x^{2}}\right. \\
& \left.-\varepsilon \operatorname{Ko} \frac{\partial C(x, t)}{\partial t}-\frac{\partial T(x, t)}{\partial t}\right] P(x, t) d x d t+\int_{0}^{t_{f}}\left[q(t)+\frac{\partial T(x, t)}{\partial x}\right] M(t) d t \\
& +\int_{0}^{t_{f}}\left[\mathrm{Bi}_{q}\left[T(x, t)-T_{a}(t)\right]-(1-\varepsilon) \operatorname{KoLuBi}_{m}\left[C(x, t)-C_{a}(t)\right]\right. \\
& \left.+\frac{\partial T(x, t)}{\partial x}\right] F(t) d t \\
& +\int_{0}^{t_{f}} \int_{0}^{L}\left[\operatorname{Lu} \frac{\partial^{2} C(x, t)}{\partial x^{2}}-\operatorname{LuPn} \frac{\partial^{2} T(x, t)}{\partial x^{2}}-\frac{\partial C(x, t)}{\partial t}\right] U(x, t) d x d t \\
& +\int_{0}^{t_{f}}\left[\operatorname{Pn} \frac{\partial T(x, t)}{\partial x}-\frac{\partial C(x, t)}{\partial x}\right] S(t) d t \\
& +\int_{0}^{t_{f}}\left[\operatorname{Bi}_{m}\left[C(x, t)-C_{a}(t)\right]+\frac{\partial C(x, t)}{\partial x}-\operatorname{Pn} \frac{\partial T(x, t)}{\partial x}\right] X(t) d t \\
& +\int_{0}^{t_{f}} \int_{0}^{L}\left[\frac{\partial^{2} T_{\varepsilon}(x, t)}{\partial x^{2}}-\operatorname{Ko} \frac{\partial C(x, t)}{\partial t}-\varepsilon \operatorname{Ko} \frac{\partial C_{\varepsilon}(x, t)}{\partial t}-\frac{\partial T_{\varepsilon}(x, t)}{\partial t}\right] Q(x, t) d x d t \\
& +\int_{0}^{t_{f}}\left[\operatorname{Bi}_{q} T_{\varepsilon}(x, t)+\operatorname{KoLuBi}_{m}\left[C(x, t)-C_{a}(t)\right]\right. \\
& \left.-(1-\varepsilon) \operatorname{KoLuBi}_{m} C_{\varepsilon}(x, t)+\frac{\partial T_{\varepsilon}(x, t)}{\partial x}\right] G(t) d t \\
& +\int_{0}^{t_{f}} \int_{0}^{L}\left[\operatorname{Lu} \frac{\partial^{2} C_{\varepsilon}(x, t)}{\partial x^{2}}-\operatorname{LuPn} \frac{\partial^{2} T_{\varepsilon}(x, t)}{\partial x^{2}}-\frac{\partial C_{\varepsilon}(x, t)}{\partial t}\right] V(x, t) d x d t \\
& +\int_{0}^{t_{f}}\left[\mathrm{Bi}_{m} C_{\varepsilon}(x, t)+\frac{\partial C_{\varepsilon}(x, t)}{\partial x}-\operatorname{Pn} \frac{\partial T_{\varepsilon}(x, t)}{\partial x}\right] Y(t) d t \\
& +\int_{0}^{t_{f}} \int_{0}^{L}\left[\frac{\partial^{2} T_{p}(x, t)}{\partial x^{2}}-\varepsilon \operatorname{Ko} \frac{\partial C_{p}(x, t)}{\partial t}-\frac{\partial T_{p}(x, t)}{\partial t}\right] R(x, t) d x d t \\
& +\int_{0}^{t_{f}}\left[\operatorname{Bi}_{q} T_{p}(x, t)-(1-\varepsilon) \operatorname{KoLuBi}_{m} C_{p}(x, t)+\frac{\partial T_{p}(x, t)}{\partial x}\right] H(t) d t
\end{aligned}
$$




$$
\begin{aligned}
& +\int_{0}^{t_{f}} \int_{0}^{L}\left[\operatorname{Lu} \frac{\partial^{2} C_{p}(x, t)}{\partial x^{2}}-\operatorname{Lu} \frac{\partial^{2} T(x, t)}{\partial x^{2}}\right. \\
& \left.-\operatorname{Lu} \operatorname{Pn} \frac{\partial^{2} T_{p}(x, t)}{\partial x^{2}}-\frac{\partial C_{p}(x, t)}{\partial t}\right] W(x, t) d x d t \\
& +\int_{0}^{t_{f}}\left[\frac{\partial T(x, t)}{\partial x}-\frac{\partial C_{p}(x, t)}{\partial x}\right] N(t) d t \\
& +\int_{0}^{t_{f}}\left[\operatorname{Bi}_{m} C_{p}(x, t)+\frac{\partial C_{p}(x, t)}{\partial x}-\frac{\partial T(x, t)}{\partial x}-\operatorname{Pn} \frac{\partial T_{p}(x, t)}{\partial x}\right] Z(t) d t
\end{aligned}
$$

The variables $P(x, t), M(t), F(t), U(x, t), S(t), X(t), Q(x, t), G(t), V(x, t), Y(t), R(x, t)$, $H(t), W(x, t), N(t)$, and $Z(t)$ represent the Lagrange multipliers. The parameter $\beta$ is a Lagrange multiplier too. By doing so, the minimization problem under constraint is transformed into another one without constraint and then the algorithm of Uzawa can be used in this case to determine the optimal control input $q(t)$ [29-33]. This algorithm needs the computation of the optimality conditions [gradient of the functional $J_{2}(q)$, and the Lagrange multiplier $\beta$ ] to be used in the iterative process. At this stage, the calculus of variations is introduced to determine the optimality conditions of $\mathscr{L}(q)$, which results, as one can see later, in the establishment of a set of variation problems, a set of adjoint problems, and the gradient of the functional $J_{2}(q)$ to be minimized.

To develop the variation problems, we assume that the unknown control $q(t)$ is perturbed by an amount $\varepsilon \Delta q(t)$, which can be written in the following form

$$
[q(t)]_{\varepsilon}=q(t)+\varepsilon \Delta q(t)
$$

Thus, the temperature $T(x, t)$, the moisture $C(x, t)$, the temperature sensitivities $T_{\varepsilon}(x, t)$ and $T_{p}(x, t)$, and the moisture sensitivities $C_{\varepsilon}(x, t)$ and $C_{p}(x, t)$ undergo small variations, whcih

$$
\begin{aligned}
& {[T(x, t)]_{\varepsilon}=T(x, t)+\varepsilon \Delta T(x, t) \quad \text { and } \quad[C(x, t)]_{\varepsilon}=C(x, t)+\varepsilon \Delta C(x, t)} \\
& {\left[T_{\varepsilon}(x, t)\right]_{\varepsilon}=T_{\varepsilon}(x, t)+\varepsilon \Delta T_{\varepsilon}(x, t) \quad \text { and } \quad\left[C_{\varepsilon}(x, t)\right]_{\varepsilon}=C_{\varepsilon}(x, t)+\varepsilon \Delta C_{\varepsilon}(x, t)} \\
& {\left[T_{p}(x, t)\right]_{\varepsilon}=T_{p}(x, t)+\varepsilon \Delta T_{p}(x, t) \quad \text { and } \quad\left[C_{p}(x, t)\right]_{\varepsilon}=C_{p}(x, t)+\varepsilon \Delta C_{p}(x, t)}
\end{aligned}
$$

Variation problems are easily derived from the direct problem (1)-(8), sensitivity problems (11)-(18), and (19)-(26) by introducing and applying the following limiting process:

$$
\lim _{\varepsilon \rightarrow 0} \frac{\mathcal{O}_{\varepsilon}-\mathcal{O}}{\varepsilon}
$$

to each equation member. The symbol $\mathcal{O}$ designates any mathematical operator, i.e., $\partial / \partial x, \partial / \partial t, \ldots$, present in the different sets of equations. After some algebraic

${ }^{1}$ Remark: $\varepsilon$ is different than $\varepsilon$. The first isa small number $(\rightarrow 0)$ used in the mathematical computations. The second has a physical sense and designates the phase conversion factor in the drying body. 
manipulations, it is easy to show that the different variation problems resulting from the variation of the control $q(t)$ are given by

$$
\begin{aligned}
& \frac{\partial \Delta T(x, t)}{\partial t}=\frac{\partial^{2} \Delta T(x, t)}{\partial x^{2}}-\varepsilon \operatorname{Ko} \frac{\partial \Delta C(x, t)}{\partial t} \quad 0<x<1, \quad t>0 \\
& \Delta T(x, t)=0 \quad 0<x<1 \quad t=0 \\
& -\frac{\partial \Delta T(x, t)}{\partial x}=\Delta q(t) \quad x=0 \quad t>0 \\
& -\frac{\partial \Delta T(x, t)}{\partial x}=\mathrm{Bi}_{q} \Delta T(x, t)-(1-\varepsilon) \mathrm{KoLuBi}_{m} \Delta C(x, t) \quad x=1 \quad t>0 \\
& \frac{\partial \Delta C(x, t)}{\partial t}=\operatorname{Lu} \frac{\partial^{2} \Delta C(x, t)}{\partial x^{2}}-\operatorname{Lu} \operatorname{Pn} \frac{\partial^{2} \Delta T(x, t)}{\partial x^{2}} \quad 0<x<1 \quad t>0 \\
& \Delta C(x, t)=0 \quad 0<x<1 \quad t=0 \\
& -\frac{\partial \Delta C(x, t)}{\partial x}+\operatorname{Pn} \frac{\partial \Delta T(x, t)}{\partial x}=0 \quad x=0 \quad t>0 \\
& -\frac{\partial \Delta C(x, t)}{\partial x}+\operatorname{Pn} \frac{\partial \Delta T(x, t)}{\partial x}=\mathrm{Bi}_{m} \Delta C(x, t) \quad x=1 \quad t>0
\end{aligned}
$$

for the direct problem (1)-(8),

$$
\begin{aligned}
& \frac{\partial \Delta T_{\varepsilon}(x, t)}{\partial t}=\frac{\partial^{2} \Delta T_{\varepsilon}(x, t)}{\partial x^{2}}-\operatorname{Ko} \frac{\partial \Delta C(x, t)}{\partial t}-\varepsilon \operatorname{Ko} \frac{\partial \Delta C_{\varepsilon}(x, t)}{\partial t} \quad 0<x<1 \quad t>0 \\
& \Delta T_{\varepsilon}(x, t)=0 \quad 0<x<1 \quad t=0 \\
& \frac{\partial \Delta T_{\varepsilon}(x, t)}{\partial x}=0 \quad x=0 \quad t>0 \\
& -\frac{\partial \Delta T_{\varepsilon}(x, t)}{\partial x}=\mathrm{Bi}_{q} \Delta T_{\varepsilon}(x, t)+\mathrm{KoLuBi}_{m} \Delta C(x, t) \\
& -(1-\varepsilon) \mathrm{KoLuBi}_{m} \Delta C_{\varepsilon}(x, t) \quad x=1 \quad t>0 \\
& \frac{\partial \Delta C_{\varepsilon}(x, t)}{\partial t}=\mathrm{Lu} \frac{\partial^{2} \Delta C_{\varepsilon}(x, t)}{\partial x^{2}}-\operatorname{Lu} \operatorname{Pn} \frac{\partial^{2} \Delta T_{\varepsilon}(x, t)}{\partial x^{2}} \quad 0<x<1 \quad t>0 \\
& \Delta C_{\varepsilon}(x, t)=0 \quad 0<x<1 \quad t=0 \\
& \frac{\partial \Delta C_{\varepsilon}(x, t)}{\partial x}=0 \quad x=0 \quad t>0
\end{aligned}
$$




$$
-\frac{\partial \Delta C_{\varepsilon}(x, t)}{\partial x}+\operatorname{Pn} \frac{\partial \Delta T_{\varepsilon}(x, t)}{\partial x}=\operatorname{Bi}_{m} \Delta C_{\varepsilon}(x, t) \quad x=1 \quad t>0
$$

for the temperature and moisture sensitivity functions with respect to $\varepsilon$, Eqs. (11-18), and finally,

$$
\begin{aligned}
& \frac{\partial \Delta T_{p}(x, t)}{\partial t}=\frac{\partial^{2} \Delta T_{p}(x, t)}{\partial x^{2}}-\varepsilon \operatorname{Ko} \frac{\partial \Delta C_{p}(x, t)}{\partial t} \quad 0<x<1 \quad t>0 \\
& \Delta T_{p}(x, t)=0 \quad 0<x<1 \quad t=0 \\
& \frac{\partial \Delta T_{p}(x, t)}{\partial x}=0 \quad x=0 \quad t>0 \\
& -\frac{\partial \Delta T_{p}(x, t)}{\partial x}=\mathrm{Bi}_{q} \Delta T_{p}(x, t)-(1-\varepsilon) \mathrm{KoLuBi}_{m} \Delta C_{p}(x, t) \quad x=1 \quad t>0 \\
& \frac{\partial \Delta C_{p}(x, t)}{\partial t}=\mathrm{Lu} \frac{\partial^{2} \Delta C_{p}(x, t)}{\partial x^{2}}-\mathrm{Lu} \frac{\partial^{2} \Delta T(x, t)}{\partial x^{2}} \\
& -\operatorname{Lu} \operatorname{Pn} \frac{\partial^{2} \Delta T_{p}(x, t)}{\partial x^{2}} \quad 0<x<1 \quad t>0 \\
& \Delta C_{p}(x, t)=0 \quad 0<x<1 \quad t=0 \\
& -\frac{\partial \Delta C_{p}(x, t)}{\partial x}+\frac{\partial \Delta T(x, t)}{\partial x}=0 \quad x=0 \quad t>0 \\
& -\frac{\partial \Delta C_{p}(x, t)}{\partial x}+\frac{\partial \Delta T(x, t)}{\partial x}+\operatorname{Pn} \frac{\partial \Delta T_{p}(x, t)}{\partial x}=\operatorname{Bi}_{m} \Delta C_{p}(x, t) \quad x=1 \quad t>0
\end{aligned}
$$

for temperature and moisture sensitivity functions with respect to Posnov number Pn, i.e., Eqs. (19-26). To complete the variation calculus, the variation of the functional to be minimized and the constraint to which it is submitted are presented as well. A simple application of the limiting process defined in Eq. (35) gives

$$
\begin{aligned}
\Delta J_{2}(q)= & -2 \sum_{i=1}^{N t} \int_{0}^{t_{f}}\left[T_{\varepsilon}\left(x_{i}, t\right) \Delta T_{\varepsilon}\left(x_{i}, t\right)+T_{p}\left(x_{i}, t\right) \Delta T_{p}\left(x_{i}, t\right)\right] d t \\
& -2 \sum_{i=1}^{N c} \int_{0}^{t_{f}}\left[C_{\varepsilon}\left(x_{i}, t\right) \Delta C_{\varepsilon}\left(x_{i}, t\right)+C_{p}\left(x_{i}, t\right) \Delta C_{p}\left(x_{i}, t\right)\right] d t
\end{aligned}
$$

for $J_{2}(q)$ and

$$
\int_{0}^{t_{f}} 2 q(t) \Delta q(t) d t
$$

for the constraint of applied control $q(t)$. Once the variation problems are established, the variation of the augmented Lagrangian functional $\Delta \mathscr{L}(q)$ can be studied 
and the optimality conditions under which this functional is stationary are deduced. This can be accomplished by writing the variation of $\Delta \mathscr{L}(q)$, i.e.,

$$
\begin{aligned}
\Delta \mathscr{L}(q)= & -2 \sum_{i=1}^{N t} \int_{0}^{t_{f}}\left[T_{\varepsilon}\left(x_{i}, t\right) \Delta T_{\varepsilon}\left(x_{i}, t\right)+T_{p}\left(x_{i}, t\right) \Delta T_{p}\left(x_{i}, t\right)\right] d t \\
& -2 \sum_{i=1}^{N c} \int_{0}^{t_{f}}\left[C_{\varepsilon}\left(x_{i}, t\right) \Delta C_{\varepsilon}\left(x_{i}, t\right)+C_{p}\left(x_{i}, t\right) \Delta C_{p}\left(x_{i}, t\right)\right] d t \\
& +\beta \int_{0}^{t_{f}} 2 q(t) \Delta q(t) d t+\ldots
\end{aligned}
$$

For the same reasons outlined above, only the first two terms are reported in the expression of $\Delta \mathscr{L}(q)$. If $\Delta T_{\varepsilon}(x, t), T_{p}(x, t), \Delta C_{\varepsilon}(x, t)$, and $C_{p}(x, t)$ are solutions to their corresponding variation problems, the stationarity conditions of $\Delta \mathscr{L}(q)$ produce the following adjoint problems [24, 27]:

$$
\begin{gathered}
-\frac{\partial P(x, t)}{\partial t}=\frac{\partial^{2} P(x, t)}{\partial x^{2}}-\operatorname{Lu} \operatorname{Pn} \frac{\partial^{2} U(x, t)}{\partial x^{2}}-\operatorname{Lu} \frac{\partial^{2} W(x, t)}{\partial x^{2}} \quad 0<x<1 \quad t<t_{f} \\
P(x, t)=0 \quad 0<x<1 \quad t=t_{f} \\
\frac{\partial P(x, t)}{\partial x}=0 \quad x=0 \quad t<t_{f} \\
-\frac{\partial P(x, t)}{\partial x}+\operatorname{LuPn} \frac{\partial U(x, t)}{\partial x}+\operatorname{Lu} \frac{\partial W(x, t)}{\partial x}=\operatorname{Bi}_{q} P(x, t) \quad x=1 \quad t<t_{f} \\
-\frac{\partial U(x, t)}{\partial t}=\operatorname{Lu} \frac{\partial^{2} U(x, t)}{\partial x^{2}}+\varepsilon \operatorname{Ko} \frac{\partial P(x, t)}{\partial t}+\operatorname{Ko}_{0} \frac{\partial Q(x, t)}{\partial t} \quad 0<x<1 \quad t>0 \\
U(x, t)=0 \quad 0<x<1 \quad t=t_{f} \\
\frac{\partial U(x, t)}{\partial x}=0 \quad x=0 \quad t<t_{f} \\
-\frac{\partial U(x, t)}{\partial x}=\operatorname{Bi}_{m} U(x, t)-(1-\varepsilon) \operatorname{KoBi}_{m} P(x, t)+\operatorname{KoBi}_{m} Q(x, t) \quad x=1 \quad t<t_{f}
\end{gathered}
$$

for the direct problem given in Eqs. (1-8),

$$
\begin{gathered}
-\frac{\partial Q(x, t)}{\partial t}=\frac{\partial^{2} Q(x, t)}{\partial x^{2}}-\operatorname{Lu} \operatorname{Pn} \frac{\partial^{2} V(x, t)}{\partial x^{2}}-2 T_{\varepsilon}(x, t) \quad 0<x<1 \quad t<t_{f} \\
Q(x, t)=0 \quad 0<x<1 \quad t=t_{f} \\
\frac{\partial Q(x, t)}{\partial x}=0 \quad x=0 \quad t<t_{f}
\end{gathered}
$$




$$
\begin{gathered}
-\frac{\partial Q(x, t)}{\partial x}+\operatorname{Lu} \operatorname{Pn} \frac{\partial V(x, t)}{\partial x}=\operatorname{Bi}_{q} Q(x, t) \quad x=1 \quad t<t_{f} \\
-\frac{\partial V(x, t)}{\partial t}=\operatorname{Lu} \frac{\partial^{2} V(x, t)}{\partial x^{2}}+\varepsilon \operatorname{Ko} \frac{\partial Q(x, t)}{\partial t}-2 C_{\varepsilon}(x, t) \quad 0<x<1 \quad t>0 \\
V(x, t)=0 \quad 0<x<1 \quad t=t_{f} \\
\frac{\partial V(x, t)}{\partial x}=0 \quad x=0 \quad t<t_{f} \\
-\frac{\partial V(x, t)}{\partial x}=\mathrm{Bi}_{m} V(x, t)-(1-\varepsilon) \mathrm{KoBi}_{m} Q(x, t) \quad x=1 \quad t<t_{f}
\end{gathered}
$$

for the temperature and moisture sensitivity functions with respect to $\varepsilon$, Eqs. (11-18), and finally,

$$
\begin{gathered}
-\frac{\partial R(x, t)}{\partial t}=\frac{\partial^{2} R(x, t)}{\partial x^{2}}-\operatorname{Lu} \operatorname{Pn} \frac{\partial^{2} W(x, t)}{\partial x^{2}}-2 T_{p}(x, t) \quad 0<x<1 \quad t<t_{f} \\
R(x, t)=0 \quad 0<x<1 \quad t=t_{f} \\
\frac{\partial R(x, t)}{\partial x}=0 \quad x=0 \quad t<t_{f} \\
-\frac{\partial R(x, t)}{\partial x}+\operatorname{Lu} \frac{\partial W(x, t)}{\partial x}=\operatorname{Bi}_{q} R(x, t) \quad x=1 \quad t<t_{f} \\
-\frac{\partial W(x, t)}{\partial t}=\operatorname{Lu} \frac{\partial^{2} W(x, t)}{\partial x^{2}}+\varepsilon \operatorname{Ko} \frac{\partial R(x, t)}{\partial t}-2 C_{p}(x, t) \quad 0<x<1 \quad t>0 \\
W(x, t)=0 \quad 0<x<1 \quad t=t_{f} \\
\frac{\partial W(x, t)}{\partial x}=0 \quad x=0 \quad t<t_{f} \\
-\frac{\partial W(x, t)}{\partial x}=\operatorname{Bi}_{m} W(x, t)-(1-\varepsilon) \operatorname{KoBi}_{m} R(x, t) \quad x=1 \quad t<t_{f}
\end{gathered}
$$

for the temperature and moisture sensitivity functions with respect to Posnov number Pn, expressed by Eqs. (19-26). The source terms appearing in these adjoint problems are located at the measurement points of temperature for $T_{\varepsilon}(x, t)$ and $T_{p}(x, t)$ and at the moisture measurements points for $C_{\varepsilon}(x, t)$ and $C_{p}(x, t)$, respectively. The same adjoint problem is obtained for $\varepsilon$ and Posnov number Pn. By taking into account (1) the hypothesis that the control $q(t)$ belongs to the Hilbert space of square integrable functions in the time domaine $0<t<t_{f}[24,27]$, and (2) the optimality 
conditions, i.e.,

$$
\Delta \mathscr{L}(q)=\Delta J_{2}(q)=\int_{0}^{t_{f}}[P(0, t)+2 \beta q(t)] \Delta q(t) d t=0
$$

we obtain the following expression for the unknown control $q(t)$ :

$$
q(t)=-\frac{1}{2 \beta} P(0, t)
$$

Now the Uzawa algorithm can be implemented to estimate iteratively the control $q(t)$ $[29,30,32,33]$. The basic steps of this algorithm are as follows.

1. Given the constraint $E$, the parameter $\varrho$, $\varepsilon_{1}$, and $\varepsilon_{2}$ three small numbers. Given an initial guess of the parameter $\beta$ and the heat flux $q(t)$, set the counters $i=0, j=0$,

2. Solve the direct and sensitivity problems, given in Eqs. (1)-(8) and (11)-(26), and compute the functional $J_{2}^{i}(q)$, given by Eq. (29),

3. Increment the counter $i=i+1$ and solve the adjoint problems given in Eqs. (63)(86). Obtain the optimal control $q(t)$ according to Eqs. (88),

4. Solve the direct and sensitivity problems, Eqs. (1)-(8) and (11)-(26), and compute the functional $J_{2}^{i}(q)$, given by Eq. (28),

5. Check the criteria $\left|J_{2}^{i}(q)-J_{2}^{i-1}(q)\right| \leq \varepsilon_{1}$ : if yes, continue; if no, go to step 3,

6. Increment the counter $j=j+1$ and compute the constraint $E^{j}$ according to Eq. (28),

7. Check the criteria $\left|E^{j}-E\right| \leq \varepsilon_{2}$; if no, modify $\beta=\beta+\varrho\left(E^{j}-E\right)$, set the counter $i=0$, and go to step 2 ,

8. Finish computation.

\section{RESULTS AND DISCUSSION}

For the design of an optimal thermal input in Luikov's equations, which makes the six parameters simultaneously easy to estimate, we examine two test cases of practical interest involving different materials: ceramics and wood. The numerical modeling of heat and moisture transfer in capillary-porous bodies requires accurate knowledge of the thermophysical properties appearing in the formulation. Table 1 summarizes the data of the two test cases examined in this article, ceramics and wood. The dimensionless exact parameters presented in this table were obtained from the themophysical properties given in [14, 15, 34]. In both cases, the dimensionless thickness of the sample was taken as $L=1$. The dimensionless experimental time, $t_{f}$, for each material, is displayed in the same table.

The direct, sensitivity, variation, and adjoint problems were solved with the finite-difference method by using an implicit scheme in time [35]. The spatial domain $0<x<L$ was discretized with 21 nodes, while 1,001 time steps were used to advance the solution from $t=0$ to $t=t_{f}$. Analytical solutions presented in [16, 17] were used to check the numerical solutions obtained with the finite-difference method. The numerical predictions of top, center, and bottom temperature and moisture distributions were compared with the analytical solution of Mikhailov, and an error less 
Table 1. Experimental data of examined test cases and estimation results with confidence intervals

\begin{tabular}{|c|c|c|c|c|c|c|c|c|c|c|}
\hline$\bullet$ & Parameter & $\mathrm{Bi}_{\mathrm{q}}$ & $\mathrm{Bi}_{\mathrm{m}}$ & $\mathrm{Lu}$ & $\mathrm{Pn}$ & Ko & $\epsilon$ & $L$ & $t_{f}$ & $q(t)$ \\
\hline \multirow[t]{10}{*}{ Ceramics } & Exact values & 2.50 & 3.33 & 0.238 & 0.084 & 49.42 & 0.200 & 1 & 10 & - \\
\hline & Initial guess & 1.00 & 1.00 & 0.01 & 0.01 & 1.00 & 0.01 & & & - \\
\hline & Upper bound & $10^{4}$ & $10^{4}$ & $10^{4}$ & $10^{4}$ & $10^{4}$ & $10^{4}$ & & & - \\
\hline & Lower bound & $10^{-4}$ & $10^{-4}$ & $10^{-4}$ & $10^{-4}$ & $10^{-4}$ & $10^{-4}$ & & & - \\
\hline & Estimated $(\sigma=0)$ & 2.500 & 3.333 & 0.2380 & 0.0840 & 49.423 & 0.2000 & & & 0.9 \\
\hline & Estimated $(\sigma \neq 0)$ & 2.509 & 3.319 & 0.238 & 0.0834 & 49.585 & 0.1999 & & & 0.9 \\
\hline & Confidence int. $( \pm)$ & 0.025 & 0.043 & 0.0015 & 0.0012 & 0.440 & 0.0019 & & & - \\
\hline & Estimated $(\sigma=0)$ & 2.50 & 3.33 & 0.238 & 0.084 & 49.42 & 0.200 & & & optimal \\
\hline & Estimated $(\sigma \neq 0)$ & 2.50 & 3.32 & 0.238 & 0.083 & 49.58 & 0.199 & & & optimal \\
\hline & Confidence int. $( \pm)$ & 0.008 & 0.015 & 0.0005 & 0.0003 & 0.181 & 0.0007 & & & - \\
\hline \multirow[t]{9}{*}{ Wood } & Exact values & 1.73 & 5.68 & 0.0085 & 0.0513 & 390.0 & 0.200 & 1 & 150 & - \\
\hline & Initial guess & 1.00 & 2.00 & 0.002 & 0.200 & 100.0 & 0.100 & & & - \\
\hline & Upper bound & $10^{4}$ & $10^{4}$ & $10^{4}$ & $10^{4}$ & $10^{4}$ & $10^{4}$ & & & - \\
\hline & Lower bound & $10^{-4}$ & $10^{-4}$ & $10^{-4}$ & $10^{-4}$ & $10^{-4}$ & $10^{-4}$ & & & - \\
\hline & Estimated $(\sigma=0)$ & - & - & - & - & - & - & & & 0.9 \\
\hline & Estimated $(\sigma \neq 0)$ & - & - & - & - & - & - & & & 0.9 \\
\hline & Estimated $(\sigma=0)$ & 1.730 & 5.681 & 0.0085 & 0.0513 & 390.00 & 0.2000 & & & optimal \\
\hline & Estimated $(\sigma \neq 0)$ & 1.733 & 5.682 & 0.0084 & 0.0 .0507 & 390.32 & 0.2000 & & & optimal \\
\hline & Confidence int. $( \pm)$ & 0.009 & 0.021 & 0.0002 & 0.0017 & 2.22 & 0.0018 & & & - \\
\hline
\end{tabular}

than $1 \%$ was observed between the two solutions for the two materials, ceramics and wood. The analytical solutions presented in the above references were derived with a constant dimensionless heat flux applied at the bottom of the sample, i.e., $x=0$. For both cases, the dimensionless heat flux was taken as $q=0.9$. Figure 2 shows analytical and numerical temperature and moisture profiles at two sensor locations, top and bottom of the sample, for the wood test case. The numerical and analytical results of Figure 2 are in good agreement, therefore the verification of the numerical finitedifference method is thus completed.

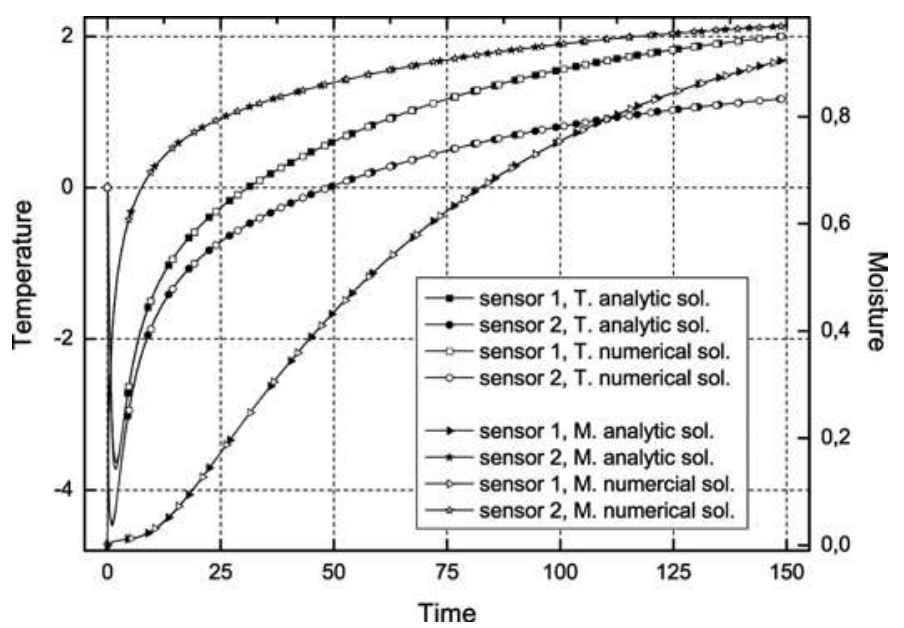

Figure 2. Comparison between analytical and numerical solutions for wood test case (T, temperature; M, moisture). 
Usually, measurement system design for a physical process involves the choice of the states to be measured and the frequency and location of measurements. Indeed, measurements at certain points in the spatial domain of the system may yield more information about the system than measurements at other points and, therefore, the accuracy of the state estimate depends on the number and locations of sensors. This idea was substantially discussed in some previous articles [14, 15].

In this work, we consider the problem of the determination of an optimal applied boundary condition for distributed parameter systems that guarantees the simultaneous estimation of Luikov's parameters from a single experiment. Since the number of sensors is generally governed by technical and economic considerations, it is desirable to locate the given number of measurement sensors at points that lead to the best estimate of the system state and with the best compromise between the technical nature and the price of sensors. Herein, we consider available the temperature and moisture measurements of two sensors installed at $x=0$ and $x=L$, respectively, for both treated materials. Installing sensors at the interface of the body (bottom and top) introduces less perturbation in the thickness of the body and makes the measurement process relatively easy to perform.

For both cases, optimal and constant heat flux profiles, the inverse problem of parameter estimation is solved with the Levenberg-Marquardt method of minimization of the least-squares norm [20, 23]. Such a method was first derived by Levenberg [36] in 1944, by modifying the ordinary least-squares norm. Later, in 1963, Marquardt [37] derived basically the same technique using a different approach. In this article, the subroutine BCLSJ from the IMSL library is used to estimate Luikov's parameters simultaneously. This subroutine solves nonlinear least-squares problems subject to bounds on the variables using a modified Levenberg-Marquardt algorithm with a user-supplied Jacobian [38]. More detailsabout this technique can be found in the specialized literature and the reference manual [39].

To examine the accuracy of the Levenberg-Marquardt method as applied to the present parameter estimation approach, we used simulated measurements containing random measurement errors with standard deviation $\sigma=0$ (errorless measurements), as well as $\sigma=0.01 T_{\max }$ and $\sigma=0.01 C_{\max }$, where $T_{\max }$ and $C_{\max }$ denote the maximum measured temperature and moisture content, respectively. Once the parameters were computed with the above computational procedure, a crude statistical analysis was performed in order to obtain estimates for the standard deviations and other quantities of interest to assess the accuracy of the estimated parameters and to analyze the influence of the chosen boundary condition [constant or optimal $q(t)$ ].

\section{Test Case 1}

Test Case 1 concerns the estimation of an optimal time-dependent heat flux $q(t)$ that makes possible the simultaneous estimation of all Luikov's parameters of ceramics from a single drying experiment with a minimum number of temperature and moisture sensors. The initial guess of the six parameters and their lower and upper bounds (constraints) used by the Levenberg-Marquardt method are displayed in Table 1. The initial guess of parameters is far enough from the exact values in comparison with the initial guess used by Datas and his co-workers [14, 15]. In a first step, the estimation of the six parameters was conducted with noiseless data and with 
a constant applied heat flux $[q(t)=0.9]$. As $q(t)$ is constant in this case, exact temperature and moisture profiles at the bottom and the top of the sample, obtained from the analytical solution of Mikhailov [16-19], were used to generate the simulated measurements.

The obtained results, shown in Table 1, match precisely the exact values (see rows 2 and 6), and no error is observed in this case. The Jacobian needed in the IMSL subroutine BCLSJ is supplied externally by the authors, and they have used a central finite-difference scheme in its computation. The perturbation epsilon used in the central finite-difference computation was taken as $10^{-5}$. By doing so, the time computation is slightly increased, but this is the price to pay for estimating these parameters. In fact, the use of the BCLSF subroutine of the IMsL library, which computes the Jacobian internally does not work in this case, and the estimation was fruitless for the chosen initial guess of parameters.

In a second step, the estimation of Luikov's parameters was conducted using noised data (temperature and moisture). The six estimated parameters and their confidence intervals are displayed in Table 1. The obtained results, over one run, are excellent, and the confidence intervals are acceptable. In this case, the computation of the Jacobian was supplied externally as in the first step.

The next step was the computation of an optimal time-dependent heat flux that makes the estimation easier. Then, the Uzawa algorithm was run with the initial guess of Luikov's parameters displayed in Table 1. The constraint $E$ over the applied heat flux $q(t)$, given by Eq. (28) and used here, was taken as 10 . It corresponds approximately to the integration of a constant $q(t)(=0.9)$ over the experimental time $t=0$ to $t=t_{f}$. The optimal heat flux profile $q(t)$ was obtained by taking the Uzawa parameters as $\alpha=0.35$ and $\varrho=0.35$. The drawback of this algorithm is the initial choice of these two parameters. Specialized mathematical literature recommends the choice of small values for these two parameters, but without giving any directives on how to do so. The optimal heat flux profile $q(t)$, displayed in Figure 3, was

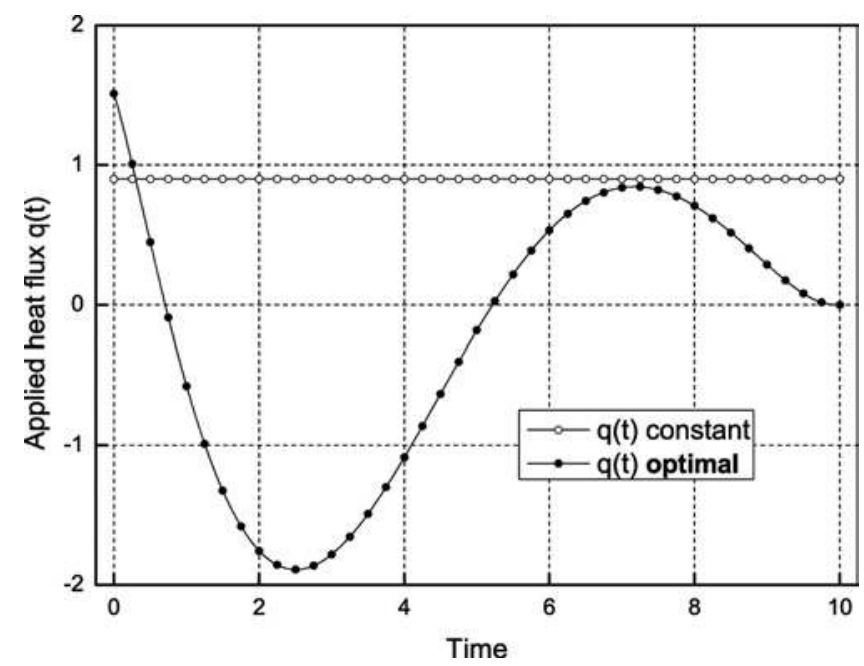

Figure 3. Constant and optimal heat flux time profile in the case of ceramics material. 
obtained in 7 iterations of $i$ counter and with approximately 31 over $j$ counter. The number $i$ represents a mean value of the iteration number at each $j$ step (see the algorithm paragraph).

Figure 3 presents the constant and optimal heat flux time profiles applied at $x=0$ (bottom of the sample). No matter the initial guess of $q(t)$, the inherent null final condition of the adjoint problem, given in Eqs. 63-86, makes the optimal $q(t)$ always null at the final time $t_{f}$ [see Eq. (88)]. As one can observe, the shape of optimal $q(t)$ is widely different than the constant one. Its maximum amplitude is twice that of the constant one.

Then this optimal heat flux profile $q(t)$ is used in the estimation procedure with noiseless and noised data. The six estimated parameters are displayed in Table 1 with their corresponding confidence intervals in the case of noiseless and noised data. For both cases, the estimation can be considered as excellent. In the case of noised data, the results obtained with a constant $q(t)$ and an optimal $q(t)$ are compared. The comparison reveals that the estimation with an optimal $q(t)$ is better than the one conducted with a constant $q(t)$. Indeed, for the same noise amplitude, the confidence intervals and the confidence region obtained with an optimal heat flux profile are significantly better. In the optimal theory control [28], the estimation accuracy for linear and nonlinear dynamic system identification is known to be maximized by the use of optimal inputs. The displayed results in Table 1 confirm this tendency.

The reduction of the least-square criterion in the Levenberg-Marquardt method, for both cases, constant and optimal $q(t)$, are compared in Figure 4. A marked difference in the decrease of the criterion is observed between the two profiles, with a large advantage to the optimal $q(t)$ profile. The number of iteration is approximately divided by 2 in the case of optimal $q(t)$. After a net decrease in the first few iterations, both profiles exhibit a stationary evolution but much longer

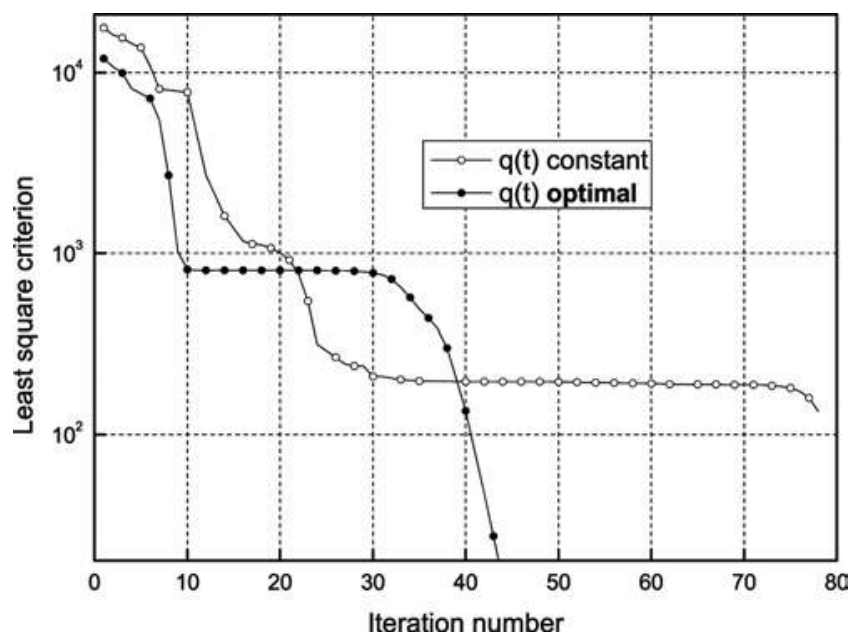

Figure 4. Evolution of the least-square criterion as a function of iteration number for both heat flux profiles; constant and optimal; ceramics test case. 
for constant $q(t)$. The stationary evolution occurs at two different levels of the least-square criterion. Then, the least-square criterion decreases drastically when the optimal $q(t)$ is used in the estimation as reported in the same Figure 4.

Figure 5 displays the sensitivity coefficients (temperature and moisture) obtained in both cases, constant and optimal $q(t)$, for the Posnov parameter Pn. By using an optimal heat flux profile, the sensitivity coefficients exhibit a great change over the duration of the experiment. After a short time from the beginning of the experiment, their amplitudes are doubled in comparison with those achieved with constant $q(t)$. This change causes a significant increase in the sensitivity of measured temperature and moisture to the unknown parameter Pn. The change from a constant to an optimal $q(t)$ influences considerably both sensitivity coefficients (temperature and moisture) in the their shape and amplitude.

Figure 6 shows the sensitivity coefficients (temperature and moisture) obtained in both cases, constant and optimal $q(t)$, for the phase conversion factor $\varepsilon$. Inspection of this figure shows that there is no substantial influence of changing the heat
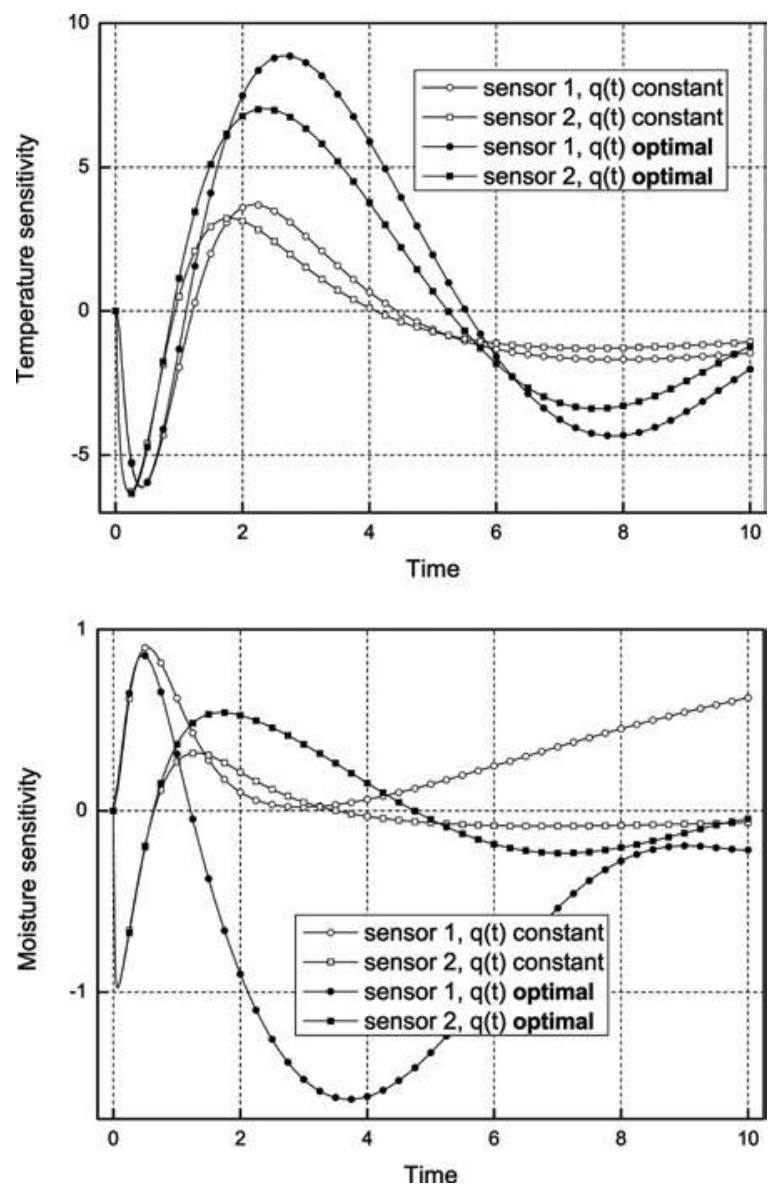

Figure 5. Sensitivity coefficients obtained with constant and optimal $q(t)$ for Pn parameter. 

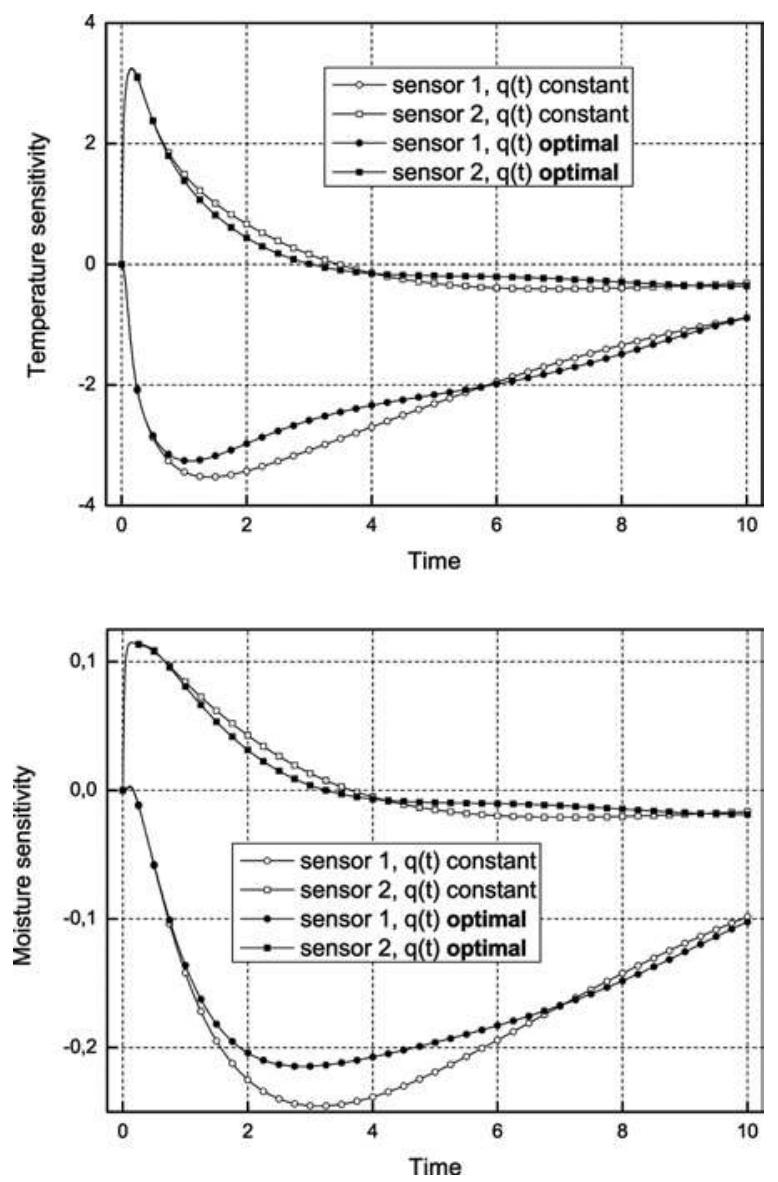

Figure 6. Sensitivity coefficients obtained with constant and optimal $q(t)$ for $\epsilon$ parameter.

flux profile on both sensitivity coefficients of $\varepsilon$. Despite a tiny change, the sensitivity coefficients exhibit the same time evolution no matter the heat flux profile used. The use of an optimal heat flux profile seems to have no influence on the moisture sensitivity coefficient with respect to $\varepsilon$ as shown by Figure 6 . Indeed, this figure presents an extremely small change of moisture sensitivity coefficient over the experiment duration.

Figure 7 compares the measured temperature and moisture profiles at the sensor locations obtained respectively with constant and optimal $q(t)$. Temperature profiles obtained with optimal $q(t)$ present a marked difference from those obtained with constant heat flux $q(t)$. As expected, the measured temperature from the sensor located at $x=0$ is more sensitive to the change of applied heat flux. On the other hand, the moisture profiles obtained with optimal $q(t)$ present a small change in comparison with the ones obtained with constant $q(t)$. Moisture measurements of sensor $2(x=L)$ do not seem to be affected by the optimal input $q(t)$, as shown by the same figure. 

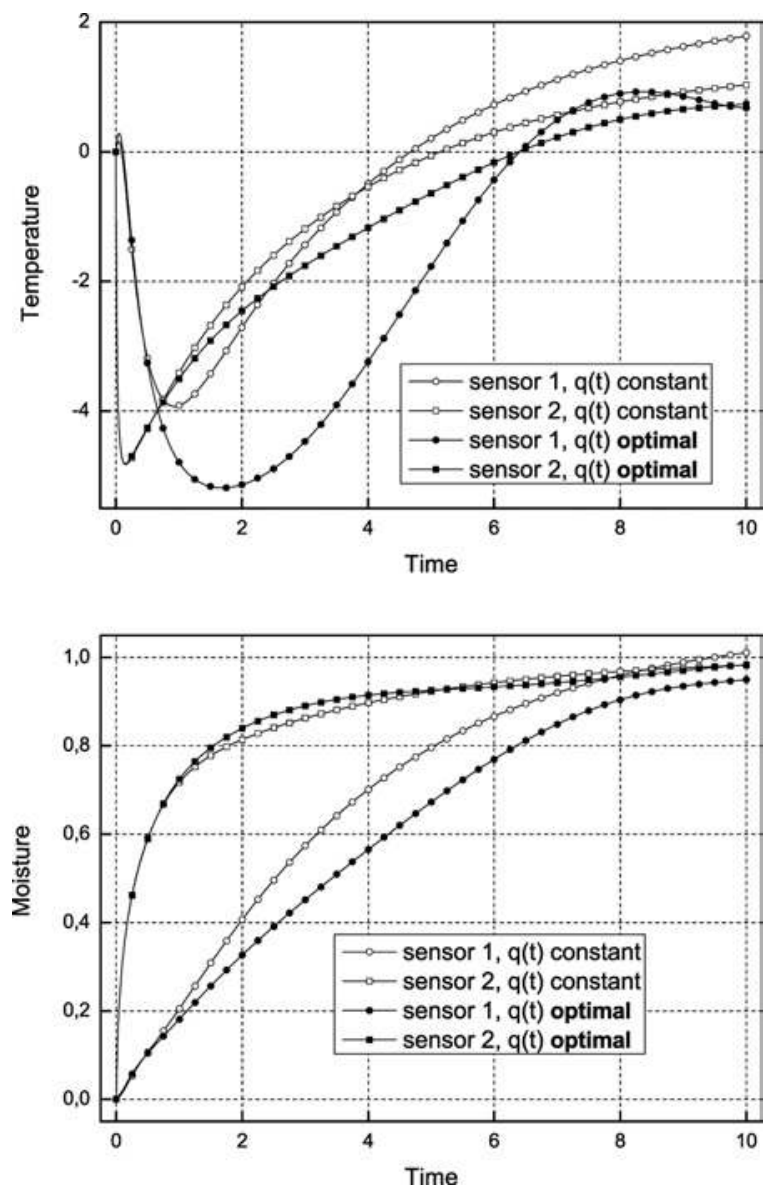

Figure 7. Measured temperature and moisture obtained with optimal and nonoptimal $q(t)$.

\section{Test Case 2}

Test Case 2 concerns the estimation of an optimal time-dependent heat flux $q(t)$ that makes possible the simultaneous estimation of all Luikov's parameters of wood from a single drying experiment with a minimum number of temperature and moisture sensors. The initial guess of the six parameters and their lower and upper bounds (contraints) used by the Levenberg-Marquardt method are displayed in Table 1.

In a first step, the estimation of the six parameters was conducted with noiseless measurements and with a constant applied heat flux $[q(t)=0.9]$. Similar to test case I, $q(t)$ is constant in this case, and exact temperature and moisture measurements at the bottom and the top of the sample, obtained from the analytical solution of Mikhailov [16-19], are used to generate the simulated measurements.

Unfortunately, with constant $q(t)(=0.9)$, the estimation fails no matter the subroutine used, BCLSJ or BCLSF, from the IMSL library. As stated before, the difference between the two subroutines is how the Jacobian, needed in the estimation procedure, is computed. The Jacobian used in the BCLSJ subroutine is supplied 
externally and computed by a central finite-difference scheme. In this case, the estimation stopped after 7 iterations when BCLSF was used and after 8 iterations when BCLSJ was used. The decrease of the least-square criterion is slightly faster with the second subroutine. In conclusion, with a constant supplied heat flux $q(t)$, the estimation does not work with the specified initial guess given in Table 1. Without checking the computation, the estimation with noised data was abandoned in the case of $q(t)$ constant.

The second step was the computation of an optimal time-dependent heat flux that makes the estimation easier. The Uzawa algorithm was run with the initial guess of Luikov's parameters displayed in Table 1. The constraint $E$ over the appliedheat flux $q(t)$, given by Eq. 28 and used here, was taken as 135. It corresponds approximately to the integration of a constant $q(t)(=0.9)$ over the experimental time $t=0$ to $t=t_{f}$. The optimal heat flux profile $q(t)$ was obtained by taking the Uzawa parameters as $\alpha=0.20$ and $\varrho=2 \times 10^{-5}$. So as not to extend the length of this article, the optimal heat flux profile $q(t)$ is not displayed here.

With an optimal heat flux profile, the estimation of Luikov's parameter with both noised and errorless measurements succeeded, and the results obtained are presented in Table 1. With errorless data, the inverse procedure retrieves precisely the exact parameters in a reasonable iteration number. The iteration process is stopped when the relative difference between two successive estimated parameters is less than or equal to $10^{-6}$.

Figure 8 displays the decrease of the least-square criterion for both cases, constant and optimal $q(t)$, when errorless measurements are used in the inverse procedure. As reported before and displayed in Figure 8, the estimation with constant $q(t)$ fails and stops after 7 iterations, while the estimation with optimal $q(t)$ works well and Luikov's parameters are retrieved after 23 iterations.

Table 1 displays the estimated parameters obtained with optimal $q(t)$ and noised data. The confidence intervals are also shown in the table and are quite

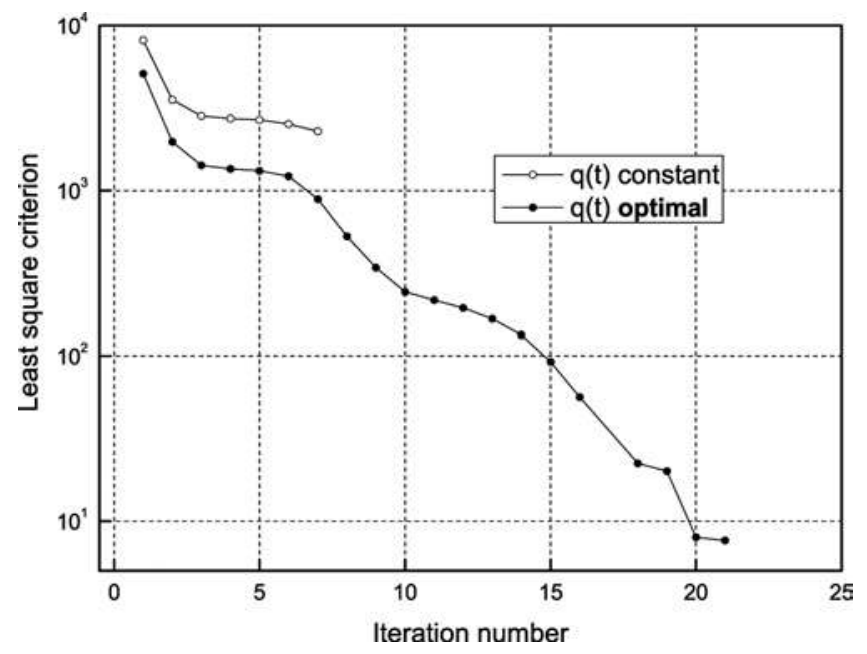

Figure 8. Least-square criterion reduction with $q(t)$ constant and optimal: wood test case. 
acceptable in comparison to the standard deviation of added white noise to temperature and moisture simulated measurements. The initial guesses of estimated parameters are as far as 1-4 orders of magnitude from the exact parameters. The use of an optimal heat flux $q(t)$ makes the simultaneous estimation of all Luikov's parameters possible.

\section{CONCLUSIONS}

A general optimization algorithm for finding an optimal input that ensures the estimation of a set of parameters in inverse heat problems has been detailed. Its presentation, derivation, and application have been conducted through the solution of an inverse heat and mass transfer problem governed by a set of linear partial differential equations. It concerns the estimation of Luikov's parameters characterizing the heat and mass transfer in a drying capillary porous material. The basic idea behind this algorithm consists of maximizing the sensitivity coefficients appearing in the estimation procedure. In other words, the performance criterion is selected in such way that the sensitivity of the measured state variables to the unknown parameters is maximized. Any given constraint on the optimal input can be taken into consideration and included in this algorithm. The estimation accuracy for system identification is known to be maximized by the use of such optimal inputs.

Two test cases were thoroughly investigated and their optimal heat inputs were obtained. The results presented show the capabilities of this method in increasing the sensitivity coefficients needed in the estimation procedure. The feasibility of estimating all of Luikov's parameters of ceramics and wood from a single drying experiment was presented and analyzed. Until now, only four parameters $\left(\mathrm{Bi}_{q}, \mathrm{Bi}_{m}, \mathrm{Lu}\right.$, and $\left.\mathrm{Ko}\right)$ among the six $\left(\mathrm{Bi}_{q}, \mathrm{Bi}_{m}\right.$, Lu, Ko, Pn, $\left.\varepsilon\right)$ were easy to estimate. By producing an optimal heat flux input, the sensitivity coefficients of parameters increases significantly, which improves the identifiability condition of all parameters and reduces the confidence intervals of the estimated parameters. Indeed,the optimal heat inputs of the test cases analyzed were noted to reduce significantly the confidence intervals of the estimated parameters.

The algorithm presented completes the different tools available in optimizing experiments for estimating heat and/or mass parameters. Among the most searched optimal conditions in the design of experiments dealing with inverse thermal problems consists of determining (1) the minimum number of sensors, (2) the best space locations of sensors, (3) the optimal frequency in collecting measurements, and (4) the optimal duration of the experiment. When one of these different tools reaches its limit, the present algorithm can be used to overcome this limitation. The results obtained by using simulated measurements with and without random errors illustrate the capabilities of the present approach to facilitate the simultaneous identification of parameters in Luikov's dimensionless linear formulation.

\section{REFERENCES}

1. A. V. Luikov, Heat and Mass Transfer in Capillary Porous Bodies, Pergamon Press, Oxford, UK, 1966. 
2. G. Kanevce, L. Kanevce, and G. S. Dulikravich, Moisture Diffusivity Estimation by Temperature Reponse of a Drying Body, in M. Tanak and G. S. Dulikravich (eds.), Inverse Problems in Engineering Mechanics, vol. II, pp. 13-22, Elsevier, Oxford, UK, 2000.

3. G. Kanevce, L. Kanevce, and G. S. Dulikravich, Influence of Boundary Conditions on Moisture Diffusivity Estimation by Temperature Response of a Drying Body, in 34th ASME Natl. Heat Transfer Conf., Pittsburgh, PA, August 2000.

4. G. Kanevce, L. Kanevce, and G. S. Dulikravich, Estimation of Drying Thermophysical Properties by Using Temperature Measurements, in H. R. B. Orlande (ed.), Inverse Problems in Engineering: Theory and Practice, vol. II, pp. 331-338, Elsevier, Rio de Janeiro, Brazil, 2002.

5. L. Kanevce, G. Kanevce, and G. S. Dulikravich, Application of Inverse Concepts to Drying, Thermal Sci., vol. 9, pp. 31-44, 2005.

6. L. Kanevce, G. Kanevce, and G. S. Dulikravich, Simultaneous Estimation of Thermophysical Properties and Heat and Mass Transfer Coefficients of a Drying Body, in M. Tanaka and G. S. Dulikravich (eds.), Inverse Problems in Engineering Mechanics, vol. III, pp. 3-12, Elsevier, Oxford, UK, 2002.

7. G. H. Kanevce, L. P. Kanevce, V. B. Mitrevski, G. S. Dulikravich, and H. R. B. Orlande, Inverse Approaches to Drying of Thin Bodies with Significant Shrinkage Effects, ASME J. Heat Transfer, vol. 129, pp. 379-386, 2007.

8. L. F. Saker and H. R. B. Orlande, Simultaneous Estimation of Spatially-Dependent Mass and Heat Transfer Coefficients of Drying Bodies, Inverse Probl. Sci. Eng., vol. 12, pp. 549561, 2004.

9. L. F. Saker, H. R. B. Orlande, C. H. Huang, and G. Kanevce, Simultaneous estimation of the Spacewise and Timewise Variations of Mass and Heat Transfer Coefficients in Drying, in Inverse Problems, Design and Optimization Symposium, Rio de Janeiro, Brazil, 2004.

10. L. F. Saker, H. R. B. Orlande, C. H. Huang, L. Kanevce, and G. Kanevce, Analysis of Different Kinds of Measurments on the Estimation of Time Dependent Mass and Heat Transfer Coefficients in Drying, in ASME International Mechanical Engineering Congress \& Exposition, Anaheim, CA, November 2004.

11. L. B. Dantas, H. R. B. Orlande, R. M. Cotta, and P. D. C. Lobo, Parameter Estimation in Moist Capillary Pourous Media by Using Temperature Measurements, in M. Tanak and G. S. Dulikravich (eds.), Inverse Problems in Engineering Mechanics, vol. II, pp. 53-62, Elsevier, Oxford, UK, 2001.

12. L. B. Dantas, H. R. B. Orlande, and R. M. Cotta, Effects of Lateral Heat Losses on the Parameter Estimation Problem in Moist Capillary Porous Media, in M. Tanak and G. S. Dulikravich (eds.), Inverse Problems in Engineering Mechanics, vol. III, pp. 13-22, Elsevier, Oxford, UK, 2001.

13. C. H. Huang and C. Y. Yeh, An Inverse Problem in Simultaneous Estimating the Biot Number of Heat and Moisture Transfer for a Porous Material, Int. J. Heat Mass Transfer, vol. 45, pp. 4643-4653, 2002.

14. H. R. B. Orlande, L. B. Dantas, and R. M. Cotta, Estimation of Dimensionless Paramaters of Luikov's System for Heat and Mass Transfer in Capillary Porous Media, Int. J. Thermal Sci., vol. 41, pp. 217-227, 2002.

15. H. R. B. Orlande, L. B. Dantas, and R. M. Cotta, An Inverse Problem of Parameter Estimation for Heat and Mass Transfer in Capillary Porous Media, Int. J. Heat Mass Transfer, vol. 46, pp. 1587-1598, 2003.

16. M. D. Mikhailov and M. N. Özisik, Unified Analysis and Solution of Heat and Mass Diffusion, John Wiley, New York, 1984.

17. M. D. Mikhailov and B. K. Shishedjiev, Temperature and Moisture Distributions During Contact Drying of a Moist Porous Sheet, Int. J. Heat Mass Transfer, vol. 18, pp. 15-24, 1975. 
18. M. D. Mikhailov, General Solutions of the Diffusion Equations Coupled at Boundary Conditions, Int. J. Heat Mass Transfer, vol. 16, pp. 2155-2164, 1973.

19. M. D. Mikhailov, General Solutions of the Coupled Diffusion Equations, Int. J. Eng. Sci., vol. 11, pp. 235-241, 1973.

20. J. V. Beck and K. J. Arnold, Parameter Estimation in Engineering and Science, John Wiley, New York, 1977.

21. E. Walter and L. Pronzato, Identification of Parametric Models from Experimental Data, Springer-Verlag, Berlin, 1997.

22. D. Uciński, Optimal Measurements Methods for Distributed Parameter System Identification, CRC Press, New York, 2005.

23. M. N. Ozisik and H. R. B. Orlande, Inverse Heat Transfer: Fundamentals and Applications, Taylor \& Francis, Philadelphia, 1999.

24. O. M. Alifanov, E. E. Artyukhin, and S. V. Rumyantsev, Extreme Methods of Solving Ill-Posed Problems and Their Applications to Inverse Heat Transfer Problems, Begell House, New York, 1995.

25. V. V. Fedorov, Theory of Optimal Experiments, Academic Press, London, 1972.

26. G. C. Goodwin and R. L. Payne, Dynamic System Identification, Academic Press, London, 1977.

27. O. M. Alifanov, Inverse Heat Transfer Problems, Springer-Verlag, Berlin, 1994.

28. R. Kalaba and K. Spingarn, Control, Identification, and Input Optimization, Plenum Press, New York, 1982.

29. M. Minoux, Mathematical Programming: Theory and Applications, John Wiley, Chichester, UK, 1986.

30. M. Chen, Excitation Optimale d'un Système Parabolic en Vue de son Identification, Ph.D. thesis, Université de Nantes, Nantes, France, 1987.

31. M. Chen and Y. Jarny, Excitation Optimale d'un Système Pareabolic en vue de l'identification, APII, vol. 24, pp. 337-355, 1990.

32. J. Burger and M. Chen, Optimal Input for the Identification of the parameters of a Nonlinear Parabolic System, SAMS, vol. 20, pp. 243-254, 1995.

33. R. Channa and M. El Bagdouri, Optimal Input for Guaranteed Identification in a Nonlinear Parabolic System, Inverse Probl. Sci. Eng., vol. 9, no. 2, pp. 95-108, 2000.

34. G. Comini and R. W. Lewis, A Numerical Solution of Two-Dimensional Problems Involving Heat and Mass Transfer, Int. J. Heat Mass Transfer, vol. 19, pp. 1387-1392, 1976.

35. M. N. Ozisik, Finite Difference Methods in Heat Transfer, CRC Press, Ann Arbor, MI, 1994.

36. K. Levenberg, A Method for the Solution of Certain Non-Linear Problems in Least Squares, Quart. Appl. Math., vol. 2, pp. 164-168, 1944.

37. D. W. Marquardt, An Algorithm for Least Squares Estimation of Non-Linear Parameters, J. Soc. Ind. Appl. Math., vol. 11, pp. 431-441, 1963.

38. J. J. Moré, The Levenberg-Marquardt Algorithm: Implementation and theory, in G. A. Watson (ed.), Numerical Analysis, Lectures Notes in Mathematics, vol. 630, pp. 105116, Springer-Verlag, Berlin, 1977.

39. IMSL, Library Edition 10.0, User's Manual, IMSL, Houston, TX, 1987. 